Mod. Phys. Lett. A 12(1997)2859.

\title{
Relativistic semiclassical wave equation and its solution
}

\author{
M. N. Sergeenko \\ The National Academy of Sciences of Belarus, Institute of Physics, \\ Minsk 220072, Belarus and \\ Gomel State University, Gomel 246699, Belarus
}

\begin{abstract}
The properties of relativistic particles in the quasiclassical region are investigated. The relativistic semiclassical wave equation appropriate in the quasiclassical region is derived. It is shown that the leading-order WKB quantization rule is the appropriate method to solve the equation obtained.

PACS number(s): 03.65.Ge, 03.65.Sq
\end{abstract}

\section{Introduction}

It is generally agreed that the full description of quantum systems is given by quantum mechanics. However, investigations by many authors show that, in fact, there exists an alternative approach. This is the approach based on the quasiclassical method.

The general form of the semiclassical description of quantum-mechanical systems has been considered in Ref. [1]. It was shown that the semiclassical description resulting from Weyl's association of operators to functions is identical with the quantum description and no information need to be lost in going from one to the another. The semiclassical approach merely becomes a different representation of the same algebra as that of the quantum mechanical system, and then the expectation values, dispersions, and dynamics of both become identical. What is more "the semiclassical description is more general than quantum mechanical description..." [1].

The quasiclassical method is based on the correspondence principle between classical functions and operators of quantum mechanics and with a real, but not necessary positive, probability density function in phase space corresponding to a particular quantummechanical state. The correspondence principle is used to derive the wave equation in quantum mechanics. In Ref. [2] this principle has been used to derive the semiclassical wave equation appropriate in the quasiclassical region. It was shown that the standard WKB method (to leading order in $\hbar$ ) is the appropriate method to solve this equation. Solution of the obtained wave equation by this method yields the exact eigenvalues for all solvable spherically symmetric potentials. The corresponding eigenfunctions have the same behavior as the asymptotes of the exact solutions.

In this work we develop the semiclassical approach for relativistic systems. We begin our analysis with the relativistic classic consideration of the problem in the HamiltonJacobi formulation. Two different versions are considered: in the first the potential is zero component of a four-vector, and in the second the potential is Lorentz-scalar. In the second version, the total energy of the system is presented by analogy with nonrelativistic case as sum of the kinetic energy and the potential energy of interaction. 
Resulting equation is written in covariant form of the relativistic one-particle equation. After that, using the correspondence principle, we derive a relativistic semiclassical wave equation appropriate in the quasiclassical region.

It is shown that the leading-order WKB quantization condition is the appropriate method to solve this equation for the spherically symmetric potentials. Solution of this equation for the Coulomb and linear potential is given. In particular, we show that unlike the existing relativistic wave equations, solution of the semiclassical wave equation for the scalar-like potential results in the eigenfunctions which are regular at the spatial origin.

\section{Relativistic Semiclassical Wave Equation}

There are several wave equations to describe the motion of relativistic particles: the Klein-Gordon equation, the Dirac equation, the Bethe-Salpeter equation, the spinless Salpeter equation, quasipotential equations. These equations can be solved exactly for some spherically symmetric potentials. However, $S$-wave solutions of these equations, for example, for the Coulomb potential diverges at the spatial origin. Wave-function divergence at the spatial origin is actually a general problem affecting relativistic wave equations. For example, the solution of the Dirac equation for the Coulomb potential for the $S$-wave states behaves as $\psi \propto r^{\sqrt{1-\alpha^{2}}-1}$ at $r \rightarrow 0$ and the divergence of the solution to the spinless Salpeter equation is much stronger: $\psi \propto r^{-4 \alpha_{s} / 3 \pi}$ [3].

Essential problem of relativistic description of physical systems is concerned with the entity of the potential. The potential is a non-relativistic conception and it has been correctly defined in classic mechanics as the function of the spatial variables. In relativistic case the potential has not been correctly defined. A serious problem is the nature of the

potential: whether it is Lorentz-vector or Lorentz-scalar or it is their mixture? This problem is especially important in hadron physics where, for the vector-like confining potential, there are no normalizable solutions [4]. In this letter we investigate both of these possibilities when the potential is the Lorentz-scalar and zero component of a 4momentum.

To derive the wave equation with the help of the correspondence principle, we need to know the corresponding classic equation. This is why we consider first classic description of relativistic particles. The classic equations are then used to derive the corresponding semiclassical wave equations.

As known, for a relativistic particle moving in the field of the spherically symmetric potential $V(r)$, the interaction is introduced according to the gauge invariant principle, $p_{\mu} \rightarrow P_{\mu}=p_{\mu}-e A_{\mu}$, where $p_{\mu}$ is the four-momentum of the particle and $A_{\mu}$ is the external field. For zero component $P_{0}=E$ this results in the total energy of the particle in the form (hereafter we basically use the system $\hbar=c=1$ )

$$
E=\sqrt{\vec{p}^{2}+m^{2}}+V(r)
$$

where $V(r)=e A_{0}$ is the static potential. Equation (1) (with the help of the correspondence principle) results in the spinless Salpeter equation [5]. For the classic equation

$\vec{p}^{2}=[E-V(r)]^{2}-m^{2}$, the same correspondence principle results in the static KleinGordon equation. 
Due to the success of the non-relativistic potential models, it can be used to scrutinize the non-relativistic method. This method has proven extremely successful for the description not only of non-relativistic systems but also of relativistic bound states. Potential models work much better than one would naively expect (see Ref. [6]). This success is somewhat puzzling in that it persists even when the model is applied to relativistic systems.

It is generally agreed, that in nonrelativistic classic mechanics, the total energy $E^{\prime}$ of a particle of mass $m$ moving in the field of the central-field potential $V(r)$ is given by equation $E^{\prime}=T+V(r)$, where $T$ is the kinetic energy of the particle; the total energy $E^{\prime}$ is the integral of motion. In relativistic case, the total energy can be written in analogous form and, what is more, the potential $V(r)$ in this case is a Lorentz-scalar.

Energy $\sqrt{\vec{p}^{2}+m^{2}}$ of relativistic particle in Eq. (1) can be presented as sum of the kinetic energy $T$ and the rest mass $m$ as $\sqrt{\vec{p}^{2}+m^{2}}=T+m$. Then equation (1) takes the form

$$
E=T+W(r)
$$

where we have introduced notation $W(r)=m+V(r)$. From nonrelativistic point of view, $W(r)$ is the potential energy shifted by constant value $m$ with respect to $V(r)$. On the other hand, from relativistic point of view, $W(r)$ can be considered as a variable mass (this topic has been discussed in series of works [7, 8, 9]). This makes sense because two ingredients of the total energy (2), the rest mass $m$ and the potential $V(r)$, are static quantities and they can be combined into one object, $W(r)$. Thus we can write for the kinetic energy $T$ of the particle of the effective mass $W(r), T=E-W(r) \equiv \vec{\pi}^{2} /[E+W(r)]$, where the squared momentum $\vec{\pi}^{2}$ is

$$
\vec{\pi}^{2}=E^{2}-W^{2}(r)
$$

Equation (3) has the form of the equation for a free relativistic particle with the effective mass $W(r)$ and can be written in the covariant form as

$$
\pi_{\mu} \pi^{\mu}-W^{2}(r)=0
$$

where $\pi^{\mu}=(E, \vec{\pi})$.

Now, we can derive the wave equations which correspond to the classic equations written above. We start with the classic problem in the Hamilton-Jacobi formulation. The static Hamilton-Jacobi equation corresponding to the classic equation $\vec{p}^{2}=[E-$ $V(r)]^{2}-m^{2}$ is

$$
\left(\frac{\partial S_{0}}{\partial r}\right)^{2}+\frac{1}{r^{2}}\left(\frac{\partial S_{0}}{\partial \theta}\right)^{2}+\frac{1}{r^{2} \sin ^{2} \theta}\left(\frac{\partial S_{0}}{\partial \varphi}\right)^{2}=[E-V(r)]^{2}-m^{2}
$$

where $S_{0}$ is the action of the system. To obtain the corresponding wave equation we present the wave function in the form $\tilde{\psi}(\vec{r})=A \exp \left[\frac{i}{\hbar} S(\vec{r})\right]$, where $A$ is the arbitrary constant. As was shown in Ref. [2], in the quasiclassical region, in representation of the wave function $\tilde{\psi}(\vec{r})$, the squared momentum operator has the form 


$$
\hat{\vec{p}}^{2}=\left(-i \hbar \frac{\partial}{\partial r}\right)^{2}+\frac{1}{r^{2}}\left(-i \hbar \frac{\partial}{\partial \theta}\right)^{2}+\frac{1}{r^{2} \sin ^{2} \theta}\left(-i \hbar \frac{\partial}{\partial \varphi}\right)^{2},
$$

which follows from the classic expression for $\vec{p}^{2}$ with the help of the correspondence principle $\frac{\partial S_{0}}{\partial q} \rightarrow-i \hbar \frac{\partial}{\partial q}, q=r, \theta, \varphi[2]$. Thus we obtain the wave equation $(\hbar=c=1)$

$$
\left[\left(-i \frac{\partial}{\partial r}\right)^{2}+\frac{1}{r^{2}}\left(-i \frac{\partial}{\partial \theta}\right)^{2}+\frac{1}{r^{2} \sin ^{2} \theta}\left(-i \frac{\partial}{\partial \varphi}\right)^{2}\right] \tilde{\psi}(\vec{r})=\left[(E-V(r))^{2}-m^{2}\right] \tilde{\psi}(\vec{r}) .
$$

Equation (7) is the second-order differential equation in canonical form. This relativistic semiclassical wave equation is closely related to the classic equation (5) and is appropriate to describe relativistic quantum systems in the quasiclassical region. Below, we solve this equation for some spherically symmetric potentials.

Let us consider now the classic equation (3). The corresponding semiclassical equation can be derived analogously to the above case. Using the operator (6), with the help of the correspondence principle we obtain the following equation

$$
\left[\left(-i \frac{\partial}{\partial r}\right)^{2}+\frac{1}{r^{2}}\left(-i \frac{\partial}{\partial \theta}\right)^{2}+\frac{1}{r^{2} \sin ^{2} \theta}\left(-i \frac{\partial}{\partial \varphi}\right)^{2}\right] \tilde{\psi}(\vec{r})=\left[E^{2}-W^{2}(r)\right] \tilde{\psi}(\vec{r}) .
$$

The correlation of the function $\tilde{\psi}(\vec{r})$ with the wave function $\psi(\vec{r})$ in case of the spherical coordinates is given by relation $\tilde{\psi}(\vec{r})=\sqrt{\operatorname{det} g_{i j}} \psi(\vec{r})$, which follows from the identity: $\int|\psi(\vec{r})|^{2} d^{3} \vec{r} \equiv \int|\psi(\vec{r})|^{2} \operatorname{det} g_{i j} d r d \theta d \varphi=1$, where $g_{i j}$ is the metric tensor (det $g_{i j}=r^{2} \sin \theta$ for the spherical coordinates).

\section{Solution of the Semiclassical Wave Equation}

Relativistic wave equations are usually solved in terms of special functions, with the help of specially developed methods or numerically. However, almost together with quantum mechanics, the appropriate method to solve the wave equation has been developed; it is general simple for all the problems, and its correct application results in the exact energy eigenvalues for all solvable potentials. This is the phase-integral method which is also known as the WKB method [10, 11].

The WKB method in its present form has been formulated to solve one-dimensional two-turning-point problems. Within the framework of the WKB method the solvable potentials mean those potentials for which the eigenvalue problem has two turning points. However the WKB method can be used to solve problems with more then two turning points.

The WKB formulas can be written differently, i.e. on the real axis 10 and in the complex plane [11. For the two-turning-point problems, the standard leading-order WKB quantization condition is defined by

$$
\int_{x_{1}}^{x_{2}} \sqrt{p^{2}(x)} d x=\pi \hbar\left(n+\frac{1}{2}\right),
$$


where $p(x)$ is the generalized momentum and $n$ is the number of zeros of the wave function inside the interval $\left[x_{1}, x_{2}\right]$. However, the most general form of the WKB solution and the quantization condition can be written in the complex plane.

The WKB solution of the one-dimensional Schrödinger's equation is searching for in the form $\psi(z)=a \exp \left[\frac{i}{\hbar} S(z)\right]$, where $a$ is the arbitrary constant and the action of the system, $S(z)$, is written as the expansion in powers of $\hbar, S(z)=S_{0}(z)+\hbar S_{1}(z)+\hbar^{2} S_{2}(z)+\ldots$. In the leading $\hbar$ approximation we have $S_{0}(z)= \pm \int^{z} p(z) d z, S_{1}(z)=i \hbar \ln \sqrt{|p(z)|}$, where $p(z)$ is the classic momentum. Thus, in this approximation, the WKB solution is

$$
\psi^{W K B}(z)=\frac{A}{\sqrt{|p(z)|}} \exp \left[\frac{i}{\hbar} \int^{z} p(z) d z\right] .
$$

In quantum mechanics, quantum numbers are determined as number of zeros of the wave function in the classically allowed region. In the complex plane, number of zeros $N$ of a function $y(z)$ inside the contour $C$ is defined by the argument's principle [12]. For the wave function $\psi(z)$, according to this principle we have

$$
\oint_{C} \frac{\psi \prime(z)}{\psi(z)} d z=2 \pi i N
$$

where $\psi \prime(z)$ is the derivative of the function $\psi(z)$. Contour $C$ is chosen such that it includes cuts between the turning points on the generalized momentum $p(z) \geq 0$ and no other singularities.

Substituting (10) into (11) we obtain the quantization condition

$$
\oint \sqrt{p^{2}(z)} d z+i \frac{\hbar}{2} \oint \frac{p \prime(z)}{p(z)} d z=2 \pi \hbar N .
$$

In the case, when $p(z)$ is a smooth function of the spatial variable and equation $p^{2}(z)=0$ has two roots (turning points), Eq. (12) results in the quantization condition

$$
\oint \sqrt{p^{2}(z)} d z=2 \pi \hbar\left(N+\frac{1}{2}\right) .
$$

In particular case $p^{2}(z)=$ const quantization condition has the form

$$
\oint \sqrt{p^{2}(z)} d z=2 \pi \hbar N \text {. }
$$

Quasiclassical method is fruitfully used not only in quantum mechanics but also in the relativistic theory 13, 14, 15. Application of the leading-order WKB quantization rule to the nonrelativistic semiclassical wave equation [2] yields the exact energy eigenvalues for all solvable spherically symmetric potentials and no further Langer-like corrections are necessary.

Equations (7) and (8) are the second-order differential equations of the Schrödinger type in canonical form. Important feature of these equations is that, for the two and more turning-point problems, they can be solved exactly by the conventional WKB method.

For the spherically symmetric potentials, Eqs. (7), (8) are separated. Angular equation for each of them is the same, 


$$
\left[\left(-i \frac{\partial}{\partial \theta}\right)^{2}+\frac{1}{\sin ^{2} \theta}\left(-i \frac{\partial}{\partial \varphi}\right)^{2}\right] \tilde{Y}(\theta, \varphi)=\vec{M}^{2} \tilde{Y}(\theta, \varphi),
$$

and the corresponding radial equations are

$$
\begin{gathered}
\left(-i \frac{d}{d r}\right)^{2} \tilde{R}(r)=\left[(E-V(r))^{2}-m^{2}-\frac{\vec{M}^{2}}{r^{2}}\right] \tilde{R}(r), \\
\left(-i \frac{d}{d r}\right)^{2} \tilde{R}(r)=\left[E^{2}-W(r)^{2}-\frac{\vec{M}^{2}}{r^{2}}\right] \tilde{R}(r) .
\end{gathered}
$$

\subsection{The angular momentum eigenvalues}

Angular equation (15) determines the squared angular momentum eigenvalues $\vec{M}^{2}$ which enter into the radial equations (16), (17). The solution of equation (15) has been obtained in Ref. [2] that gives for $\vec{M}^{2}$,

$$
\vec{M}^{2}=\left(l+\frac{1}{2}\right)^{2}, \quad l=0,1,2, \ldots
$$

This same result can be obtained in the complex plane with the help of the quantization condition (13). It is interesting from the methodological point of view to bring here the calculation of $\vec{M}^{2}$ in the complex plane because this calculation is shorter and beautiful.

Separation of the angular Eq. (15) results in two one-dimensional equations,

$$
\begin{gathered}
\left(-i \frac{d}{d \theta}\right)^{2} \tilde{\Theta}(\theta)=\left(\vec{M}^{2}-\frac{M_{z}^{2}}{\sin ^{2} \theta}\right) \tilde{\Theta}(\theta), \\
\left(-i \frac{d}{d \varphi}\right)^{2} \tilde{\Phi}(\varphi)=M_{z}^{2} \tilde{\Phi}(\varphi),
\end{gathered}
$$

The WKB quantization condition (14) which is appropriate to the angular equation (20) $\left[p(\varphi)=M_{z}\right]$, gives $M_{z}=m, m=0,1,2, \ldots$ The corresponding quasiclassical solution is

$$
\tilde{\Phi}_{m}(\varphi)=A e^{i m \varphi}+B e^{-i m \varphi},
$$

where $A$ and $B$ are the arbitrary constants.

The WKB quantization condition (13) appropriate to equation (19) is

$$
I=\oint_{C} \sqrt{\vec{M}^{2}-\frac{M_{z}^{2}}{\sin ^{2} \theta}} d \theta=2 \pi\left(n_{\theta}+\frac{1}{2}\right), \quad n_{\theta}=0,1,2, \ldots
$$

To calculate integral (22) (as other hereafter) we use the method of stereographic projection. This means that, instead of integration about a contour $C$ enclosing the classical turning points, we exclude the singularities outside the contour $C$, i.e., at $\theta=0$ and $\infty$ in this particular case. Excluding these infinities we have, for the integral (22), $I=I_{0}+I_{\infty}$. Integral $I_{0}=-2 \pi M_{z}$, and $I_{\infty}$ is calculated with the help of the replacement $z=e^{i \theta}$ that gives $I_{\infty}=2 \pi \sqrt{\vec{M}^{2}}$. Therefore $I=2 \pi\left(\sqrt{\vec{M}^{2}}-M_{z}\right)$ and we obtain eigenvalues (18). 
Equation (18) represents the squared angular momentum eigenvalues in the quasiclassical region which are different from the known ones $l(l+1)$ obtained from the solution of the Klein-Gordon equation. The centrifugal term in the radial equations (16), (17) has the form $\left(l+\frac{1}{2}\right)^{2} / r^{2}$ for all spherically symmetric potentials $V(r)$ which is not equal to zero at $l=0$. The WKB solution corresponding to the eigenvalues (18) has the correct asymptotic behavior at $\theta \rightarrow 0$ and $\pi$ for all values of $l$ and agrees with the asymptote of the spherical functions $Y_{l m}(\theta, \varphi)$ [2]. It is important to emphasize that the squared angular momentum eigenvalues (18) have obtained in our approach from the solution of the angular semiclassical equation (15) in the framework of the same quasiclassical method.

\subsection{The Coulomb potential $V(r)=-\alpha / r$}

Consider several examples to show the efficiency of the semiclassical approach in relativistic theory. Let us deal with first the classic problem for the Coulomb potential, i.e. the radial semiclassical equation (16). The quantization condition (13) appropriate to Eq. (16) with the Coulomb potential is

$$
\oint_{C} \sqrt{E^{2}-m^{2}+\frac{2 \alpha E}{r}-\frac{\Lambda^{2}}{r^{2}}}=2 \pi\left(n_{r}+\frac{1}{2}\right),
$$

where $\Lambda^{2}=\left(l+\frac{1}{2}\right)^{2}-\alpha^{2}$ and a contour $C$ encloses the classical turning points $r_{1}$ and $r_{2}$. Using the method of stereographic projection, we should exclude the singularities outside the contour $C$, i.e. at $r=0$ and $\infty$. Excluding these infinities we have, for the integral (23), $I=2 \pi\left(\alpha E / \sqrt{-E^{2}+m^{2}}-\Lambda\right)$, and for the energy eigenvalues this gives

$$
E_{n}=\frac{m}{\sqrt{1+\frac{\alpha^{2}}{\left(n_{r}+\frac{1}{2}+\Lambda\right)^{2}}}} .
$$

Equation (24) coincides with the one obtained from the solution of the Klein-Gordon equation with the Coulomb potential by known exact method.

Now, let us deal with the semiclassical wave equation (17). The quantization condition (13) appropriate to Eq. (17) with the scalar-like Coulomb potential is

$$
\oint_{C} \sqrt{E^{2}-\left(m-\frac{\alpha}{r}\right)^{2}-\frac{\left(l+\frac{1}{2}\right)^{2}}{r^{2}}} d r=2 \pi\left(n_{r}+\frac{1}{2}\right) .
$$

The phase-space integral (25) is calculated analogously to the above case in closed form that results in the energy eigenvalues

$$
E_{n}=m \sqrt{-\frac{\alpha^{2}}{\left[n_{r}+\frac{1}{2}+\sqrt{\left(l+\frac{1}{2}\right)^{2}+\alpha^{2}}\right]^{2}}+1} .
$$

Formula (26) can be written in apparent relativistic form, $E_{n}^{2}=m^{2}+p_{n}^{2}$, where $p_{n}$ is given by equation

$$
p_{n}=\frac{i \alpha m}{n_{r}+\frac{1}{2}+\sqrt{\left(l+\frac{1}{2}\right)^{2}+\alpha^{2}}} .
$$


The radial quasiclassical eigenfunctions inside the region of classic motion $\left[r_{1}, r_{2}\right]$ are written with the help of the Eq. (10)

$$
\tilde{R}^{W K B}(r)=\frac{A}{\sqrt{|p(r)|}} \cos \left(\int_{r_{1}}^{r} \sqrt{p^{2}(r)} d r-\frac{\pi}{4}\right) .
$$

So far, as $|p(r)| \simeq \frac{\Lambda}{r}$ at $r \rightarrow 0$, this gives, for the WKB solution in the representation of the wave function $\psi(\vec{r}), R(r)=\tilde{R}^{W K B}(r) / r \propto r^{\Lambda} / \sqrt{r}$. Because $\Lambda=\sqrt{\left(l+\frac{1}{2}\right)^{2}+\alpha^{2}}$, for the $S$-wave states, this results in $R(r) \propto r^{\alpha}$, i.e. $R(r)$ is regular at small $r$. We obtain this result because the potential in Eq. (17) is scalar-like. Note that in the case of the Klein-Gordon equation the radial $S$-wave function $R(r)$ diverges at $r \rightarrow 0$, i.e. $R(r) \propto r^{-\alpha}$.

The quasiclassical solution (28) can be written in the form of a standing wave. For this note that the hermiticity of the operator (6) implies the adiabatically slow alteration of the derivatives $\frac{\partial S_{0}}{\partial q}, q=r, \theta, \varphi$, i.e. $\frac{\partial S_{0}}{\partial q} \simeq$ const [2]. This condition anticipates the final result, i.e. discrete constant eigenvalues $p_{n}$ of the operator (6). Integration of equation $\frac{\partial S_{0}}{\partial r} \simeq$ const gives, for the action $S_{0}, S_{0}(r)=\left|p_{n}\right| r+$ const, where $p_{n}$ is given by Eq. (27) (for this problem). In the region of classic motion, where $p(r)>0$, the solution has the form of a standing wave

$$
\tilde{R}_{n}(r)=C \cos \left(\left|p_{n}\right| r-\chi_{1}-\frac{\pi}{4}\right)
$$

where $\chi_{1}$ is the value of the phase integral (25) at the turning point $r_{1}$. Analogous solutions can be written for other one-dimensional equations obtained after separating of Eq. (8).

Thus we obtain two results for the Coulomb potential by the WKB method: the known exact eigenvalues for spinless particles (24) which coincide with those obtained from the solution of the Klein-Gordon equation and the other ones (26) obtained from the solution of the semiclassical equation (8) with the scalar-like Coulomb potential. We compare two relativistic formulas (24) and (26) for the energy eigenvalues of an electron moving in the field of the static spherically symmetric Coulomb potential in Table 1. 
Table 1. Energy levels of the hydrogen atom

\begin{tabular}{ccccc}
\hline \hline$l$ & $n_{r}$ & $E_{n}^{N R}$ & $E_{n}^{K G}$ & $E_{n}^{S C}$ \\
\hline & & & & \\
0 & 0 & -13.6155700 & -13.6164800 & -13.6143000 \\
1 & 0 & -3.4038930 & -3.4039190 & -3.4038440 \\
0 & 1 & -3.4038930 & -3.4040400 & -3.4037230 \\
1 & 1 & -1.5128410 & -1.5128520 & -1.5128250 \\
2 & 1 & -0.8509732 & -0.8509756 & -0.8509693 \\
2 & 2 & -0.5446228 & -0.5446243 & -0.5446208 \\
3 & 2 & -0.3782103 & -0.3782109 & -0.3782095 \\
4 & 2 & -0.2778688 & -0.2778690 & -0.2778684 \\
4 & 3 & -0.2127433 & -0.2127435 & -0.2127430 \\
\hline \hline
\end{tabular}

In Table $1 E_{n}^{N R}$ denotes the eigenenergies obtained from solution of the Schrödinger equation and $E_{n}^{K G}$ are the energy eigenvalues obtained from the Klein-Gordon equation, $l$ and $n_{r}$ are the orbit and radial quantum number, respectively. Eigenenergies $E_{n}^{S C}$ have been calculated with the help of Eq. (26).

As is well known, the energy spectrum resulting from the Klein-Gordon equation with the Coulomb potential contains a fine-structure effect giving an over-estimation of the fine-structure level spreading, while the result from non-relativistic Schrödinger equation does not [10]. In our case, the eigenvalues $E_{n}^{S C}$ are lower than $E_{n}^{N R}$ and $E_{n}^{K G}$, hereby $E_{n}^{S C}$ are closer to non-relativistic ones. Fine-structure term which follows from $E_{n}^{S C}$ is

$$
\Delta E_{n}^{S C}=\frac{m \alpha^{4}}{2 n^{3}}\left(\frac{1}{l+\frac{1}{2}}-\frac{1}{4 n}\right)
$$

where $n=n_{r}+l+1$. This term is different from the corresponding correction which follows from $E_{n}^{K G}$ by sign and the second term.

\subsection{The linear potential $V(r)=\kappa r$}

The linear potential is one of the special interest in hadron physics. In relativistic case, this potential results in the linear Regge trajectories which observed at experiment. In relativistic potential models of quarkonia based on a Dirac-type equation with a local potential is a sharp distinction between a linear potential $V$ which is vector-like and one which is scalar-like. There are normalizable solutions for a scalar-like $V$ but not for a vector-like $V$ [4]. No other problems arise and no difficulties encountered with the numerical solution if the confinement potential is purely scalar-like. It was shown in many works that the effective interaction has to be scalar in order to confine particles inside the hadrons (see, for example, Refs. [4, 16]).

In relativistic theory the linear potential represents the so-called "insoluble" problem with four turning points. This problem is insoluble also from the viewpoint of the conventional WKB method. However, this problem can be solved by the WKB method in the complex plane. In the semiclassical equation (17), the potential is a scalar-like. Consider 
this equation for a two-particle system of equal masses interacting by means of the linear potential. The radial semiclassical equation (17) is then:

$$
\left(-i \frac{d}{d r}\right)^{2} \tilde{R}(r)=\left[\frac{E^{2}}{4}-(m+\kappa r)^{2}-\frac{\left(l+\frac{1}{2}\right)^{2}}{r^{2}}\right] \tilde{R}(r),
$$

From four turning points of this problem, two of them, $r_{1}$ and $r_{2}$, lie in nonphysical region $r<0$, and the other two, $r_{3}$ and $r_{4}$ lie in the physical region $r>0$. To calculate the phase-space integral in the complex plane we chose a contour $C$ enclosing the cuts (and, therefore, zeros of the wave function) at $r>0$ and $r<0$ between the turning points $r_{1}$, $r_{2}$ and $r_{3}, r_{4}$, respectively. Thus, the quantization condition appropriate to Eq. (31) is:

$$
I=\oint_{C} \sqrt{\frac{E^{2}}{4}-(m+\kappa r)^{2}-\frac{\left(l+\frac{1}{2}\right)^{2}}{r^{2}}} d r=4 \pi\left(n_{r}+\frac{1}{2}\right),
$$

where $n_{r}$ denotes zeros of the wave function at $r>0$.

Outside the contour $C$, the problem has two singularities, i.e. at $r=0$ and $\infty$. Using the method of stereographic projection, we have, for the integral $(32), I=I_{0}+I_{\infty}$, where $I_{0}=-2 \pi\left(l+\frac{1}{2}\right)$ and the integral $I_{\infty}$ is calculated with the help of the replacement of variable, i.e. $z=\frac{1}{r}$, that gives $I_{\infty}=2 \pi E^{2} /(8 \kappa)$. Therefore, for $E_{n}^{2}$, we obtain

$$
E_{n}^{2}=8 \kappa\left(2 n_{r}+l+\frac{3}{2}\right)
$$

which is very similar to that of a harmonic oscillator-type Hamiltonian.

It is an experimental fact that the dependence $E_{n}^{2}(l)$ is linear for light mesons (linear Regge trajectories [17]). However, at present, the best way to reproduce the experimental masses of particles is to rescale the entire spectrum assuming that the masses $M$ of the mesons are expressed by the relation [16]

$$
M_{n}^{2}=E_{n}^{2}-C^{2}
$$

where $E_{n}$ is given by (33) and $C$ is a constant energy (free parameter). Relation (34) is used to shift the spectra and appears as a means to simulate the effects of unknown structure approximately.

However, the required shift of the spectra naturally follows from the solution of the semiclassical equation (17). To describe the Regge trajectories observed at experiment one needs to take into account the week coupling effect. In this case the potential includes the Coulomb-like term, $-\alpha_{s} / r$, i.e. the scalar-like potential is $V(r)=-\alpha_{s} / r+\kappa r$ (the funnel potential). For light mesons one may expect that the $q \bar{q}$ bound states will feel only the linear part of the potential which gives the main contribution to the binding energy. We thus assume that the Coulomb term can be considered as a small perturbation. Then we obtain instead of (33)

$$
E_{n}^{2}=8 \kappa\left(2 n_{r}+l-\alpha_{s}+\frac{3}{2}\right)
$$

Equation (35) does not require any additional free parameter. We obtain the necessary shift with the help of the term $-8 \kappa \alpha_{s}$ which is the result of interference of the 
Coulomb and linear terms of the interquark potential. Note that we obtain the correct sign (minus) for this term only in the case of the scalar-like potential. Equation (35) gives a good description of mass of light vector mesons for the typical value $\kappa \simeq 0.14 \mathrm{GeV}^{2}$, i.e. reproduces the linear dependence of angular momentum $l$ on $E^{2}$ that is in agreement with the experimental data for light mesons [17.

\section{Conclusion}

In this letter, we have developed the semiclassical approach to the description of relativistic systems. In our analysis we have started with the relativistic classic consideration of the problem in the Hamilton-Jacobi formulation. Two different possibilities have been considered: in the first the potential is the zeroth component of a four-vector and in the second, the potential is the Lorentz-scalar.

The main result is the derivation of the relativistic semiclassical wave equation [(7) for the vector-like potential and (8) for the scalar-like potential] appropriate in the quasiclassical region. This equation is the second-order differential equation in canonical form (without first derivatives). In case of the scalar-like potential, the resulting equation has been written in covariant form. Unlike the Klein-Gordon equation, the semiclassical one (in case of the vector-like potential) results in another integral of motion, i.e. the squared angular momentum $\vec{M}^{2}=\left(l+\frac{1}{2}\right)^{2} \hbar^{2}$. This is same squared angular momentum we have obtained also for the semiclassical equation with the scalar-like potential.

The squared angular momentum eigenvalues $\vec{M}^{2}=\left(l+\frac{1}{2}\right)^{2} \hbar^{2}$ have been obtained in our approach in a natural way from the solution of the angular semiclassical equation (15) in the framework of the same WKB method that gives the justification of the Langer correction as that to the squared angular momentum eigenvalues. This means that the centrifugal term in the radial semiclassical equation has the form $\left(l+\frac{1}{2}\right)^{2} / r^{2}$ for any spherically symmetric potential $V(r)$.

Important feature of the semiclassical wave equation is that it can be solved by the WKB method. We have shown that the standard leading-order WKB quantization condition reproduces the exact eigenvalues for two-turning-point problems. Besides, this equation can be solved by the WKB method for more then two turning-point problems.

To show efficiency of the semiclassical approach in relativistic theory we have considered several examples. In particular, we have obtained two results for the Coulomb potential by the WKB method: the known exact result for spinless particles (24) which coincides with one obtained from solution of the Klein-Gordon equation and another result (26) obtained from the solution of the semiclassical equation (8) for the scalar-like Coulomb potential. We have shown that, unlike the known relativistic wave equations for the Coulomb potential, the semiclassical one for the scalar-like Coulomb potential has the regular solution at the spatial origin. Conception of the scalar-like potential is especially important in hadron physics. The solution of equation (8) for light $q \bar{q}$ states with the funnel potential has allowed us to reproduce the linear dependence $E^{2}(l)$ and obtain apparent form of the shift parameter $C^{2}, C^{2}=-8 \alpha \kappa$.

Acknowledgment. This work was supported in part by the Belarusian Fund for Fundamental Researches. 


\section{References}

[1] S. P. Misra and T. S. Shankara, J. Math. Phys. 9, 299 (1968).

[2] M. N. Sergeenko, Phys. Rev. A 53(6) (1996) 3798.

[3] L. Durand, Phys. Rev. D 32, 1257 (1985).

[4] J. Sucher, Phys. Rev. D 51, 5965 (1995).

[5] E. E. Salpeter, Phys. Rev. 87, 328 (1952).

[6] W. Lucha and F. F. Schöberl, Int. J. Mod. Phys. A7(26), 6431 (1992); W. Lucha, H. Rupprecht and F.F. Schöberl, Phys. Rev. D 46, 1088 (1992).

[7] S. Ono, Phys. Rev. D 26, 2510 (1982);

[8] F. Ravndal, Phys. Lett. B 113, 57 (1982);

[9] T. A. Dullemond, E. Rijken van Beveren, Nuovo Cim., A 80, 401 (1984); A. K. Gorbatzevich and L. M. Tomilchik, Preprint 415, IP ASB, Minsk 1986.

[10] L. Schiff: Quantum mechanics (2nd edition McGraw-Hill, New York-Toronto-London, 1955).

[11] N. Fröman and P. O. Fröman, JWKB Approximation: Contributions to the Theory (North Holland, Amsterdam, 1965); J. Heading, An Introduction to Phase-Integral methods (John Wiley \& Sons, Inc. New York, 1962).

[12] G. A. Korn, T. M. Korn: Mathematical Handbook (2nd, enlarged and revised edition (McGraw-Hill, New York-San Francisco-Toronto-London-Sydney, 1968).

[13] J. S. Kang and H. J. Schnitzer, Phys. Rev. D 12, 841 (1975).

[14] M. N. Sergeenko, Z. Phys. C 64, 315 (1994); Phys. At. Nucl. 56, 365 (1993).

[15] S. Kruglov and M. N. Sergeenko, Mod. Phys. Lett. A, 1997 (to be printed).

[16] C. Semay and R. Ceuleneer, Phys. Rev. D 48, 4361 (1993).

[17] P. Collins, E. Squires: An Introduction to Regge Theory and High Energy Physics (Cambridge, University Press, Cambridge, 1977); J. Bjorken: SLAC-PUB-6463. 1994. 